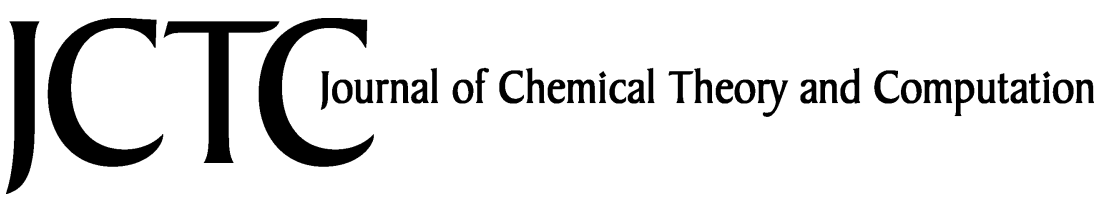

\section{Performance of CASPT2 and DFT for Relative Spin-State Energetics of Heme Models}

\author{
Steven Vancoillie, ${ }^{\dagger}$ Hailiang Zhao, ${ }^{\dagger}$ Mariusz Radon, ${ }^{\ddagger}$ and Kristine Pierloot ${ }^{*}{ }^{\dagger}$ \\ Department of Chemistry, University of Leuven, Celestijnenlaan 200F, \\ B-3001 Heverlee-Leuven, Belgium and Faculty of Chemistry, Jagiellonian University, \\ ul. Ingardena 3, 30-060 Kraków, Poland
}

Received October 26, 2009

\begin{abstract}
The accuracy of the relative spin-state energetics of three small Fe" or Fe"ll heme models from multiconfigurational perturbation theory (CASPT2) and density functional theory with selected functionals (including the recently developed M06 and M06-L functionals) was assessed by comparing with recently available coupled cluster results. While the CASPT2 calculations of spin-state energetics were found to be very accurate for the studied Fe $\mathrm{F}^{\text {III }}$ complexes (including $\mathrm{FeP}(\mathrm{SH})$, a model of the active site of cytochrome $\mathrm{P} 450$ in its resting state), there is a strong indication of a systematic error (around $5 \mathrm{kcal} / \mathrm{mol}$ ) in favor of the high-spin state for the studied Fell complexes (including FeP(Im), a model of the active site of myoglobin). A larger overstabilization of the high-spin states was observed for the M06 and M06-L functionals, up to 22 and $11 \mathrm{kcal} / \mathrm{mol}$, respectively. None of the tested density functionals consistently provides a better accuracy than CASPT2 for all model complexes.
\end{abstract}

\section{Introduction}

Because of their important role in biological systems as the active centers or prosthetic groups of heme proteins ${ }^{1}$ iron porphyrins have over the years received a lot of attention. The elucidation of both the geometric and the electronic structures of these compounds is of paramount importance for the detailed understanding of the complex mechanisms of biological systems. ${ }^{2}$ An important aspect of iron porphyrins is that during the catalytic processes the spin state of the central iron changes. Both $3 \mathrm{~d}^{6} \mathrm{Fe}$ (II) and $3 \mathrm{~d}^{5} \mathrm{Fe}$ (III) porphyrins can access low-spin (LS; singlet or doublet), intermediate-spin (IS; triplet or quartet), and high-spin (HS; quintet or sextet) states. Consequently, a good description of the relative spin-state energetics is required for any method aimed at achieving a good accuracy for describing hemeligand bond formation.

For the quantum chemical treatment of iron porphyrins, the methods that are most readily employed are either density functional theory (DFT) or multiconfigurational second-order

\footnotetext{
* Corresponding author fax: +32 $16 \quad 32 \quad 79$ 92; e-mail: Kristin.Pierloot@chem.kuleuven.be.

$\dagger$ University of Leuven.

*agiellonian University.
}

perturbation theory (CASPT2). The former is dominant in the area of bioinorganic molecules because it accounts for electron correlation at a low computational cost, allowing for treatment of large molecules. Unfortunately, the results are significantly dependent on the functional, especially for predicting relative spin-state energetics in transition-metal complexes. ${ }^{3-25}$ The CASPT2 method on the other hand is the only feasible ab initio alternative for DFT in cases of relatively large transition-metal compounds. This method was shown to outperform DFT $^{20,22}$ with several traditional (GGA or hybrid) functionals in two comparative studies of the HSLS splittings of a number of six-coordinated ferrous compounds. ${ }^{20,22}$ However, there are also indications that CASPT2 may in fact significantly overstabilize higher with respect to lower spin states at least in some (critical) cases. A typical example is ferrous porphin $\mathrm{FeP}(\mathrm{P}=$ porphin $)$, for which CASPT2 is unable to predict the correct ${ }^{3} \mathrm{~A}_{2 g}$ ground state. Instead, $\mathrm{HS}^{5} \mathrm{~A}_{1 g}$ is the calculated ground state, $5 \mathrm{kcal} / \mathrm{mol}$ below the ${ }^{3} \mathrm{~A}_{2 g}$ state. ${ }^{23,26}$ In this respect, it should be noted that the CASPT2 method, in its present implementation in the MOLCAS 7.x software, ${ }^{27}$ already includes in its zeroth-order Hamiltonian $\hat{H}^{(0)}$ an ionization potential-electronic affinity (IPEA) shift technique to properly discriminate the HS and LS states. Without this shift, the error on the 


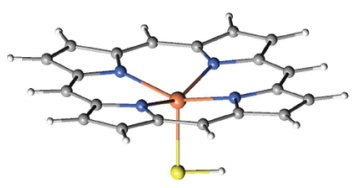

(a) $\mathrm{FeP}(\mathrm{SH})$

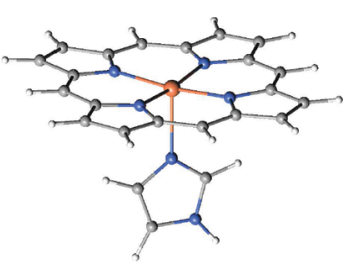

(b) $\mathrm{FeP}(\mathrm{Im})$
Figure 1. Molecular structures of heme models.

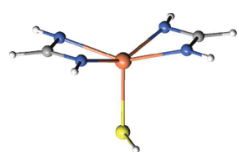

(a) model 1

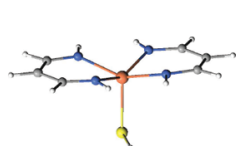

(b) model 2

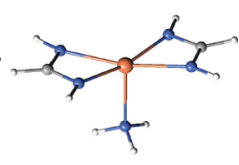

(c) model 3
Figure 2. Molecular structures of the small models.

${ }^{5} \mathrm{~A}_{1 g}-{ }^{3} \mathrm{~A}_{2 g}$ gap in FeP is significantly larger, up to $10 \mathrm{kcal} /$ mol. $^{26}$ In the original implementation of CASPT2 in MOLCAS, the IPEA shift was absent. However, already in one of the first systematic test studies of the method it was shown that the original $\hat{H}^{(0)}$ would systematically favor HS over LS states. ${ }^{28,29}$ A first remedy was formulated in the so-called $g 1, g 2$, and $g 3$ modifications of $\hat{H}^{(0)},{ }^{30}$ but later the IPEA-modified $\hat{H}^{(0)}$ was introduced ${ }^{31}$ and became the standard zeroth-order Hamiltonian in MOLCAS 6.4. The standard IPEA shift was set to 0.25 au, based on systematic tests of dissociation, ionization, and excitation energies in atoms and simple molecules. However, in a recent CASPT2 study on spin-cross-over complexes with $\mathrm{Fe}(\mathrm{II}) \mathrm{N}_{6}$ architecture it was suggested ${ }^{32}$ that a shift of 0.25 au is in fact too small to properly describe the adiabatic HS-LS gap in these systems, and a shift of $0.5-0.7$ au was proposed instead.

In a recent study by Oláh and Harvey, the performance of several popular DFT functionals for treating NO bonding to heme groups with ferric or ferrous iron was investigated. ${ }^{25}$ To this end, DFT calculations were performed on FeP(Im) and $\mathrm{FeP}(\mathrm{SH})$ (Figure 1). The latter models the cystein-ligated $\mathrm{Fe}(\mathrm{III})$ heme group which is commonly found in cytochrome $\mathrm{P} 450$ s and other hemoproteins, while the former serves as a model for histidine-ligated $\mathrm{Fe}(\mathrm{II})$ porphyrins found in the active site of many enzymes, e.g., in myoglobin. In order to assess the accuracy of the DFT functionals for the spin-state energetics a series of benchmark calculations was performed by means of the $\operatorname{CCSD}(\mathrm{T})$ method and different basis sets. Because this was not possible for the $\mathrm{FeP}(\mathrm{SH})$ and $\mathrm{FeP}(\mathrm{Im})$ molecules, three smaller model systems were used using two chelating amidine ligands instead of the full porphyrin ring (Figure 2).

The main purpose of the present study is to test the accuracy/error of CASPT2 for describing the spin-state energetics in ferrous and ferric porphyrins. To this end, CASSCF/CASPT2 calculations with different basis sets were first performed on the small heme models introduced by Oláh and Harvey so as to compare to their $\operatorname{CCSD}(\mathrm{T})$ benchmark results. The role played by the IPEA shift in $\hat{H}^{(0)}$ was investigated by performing test calculations where this shift was increased to $0.5 \mathrm{au}$. As a second point of interest, in a continued search for improved exchange-correlation func- tionals for the property at hand we decided to include in this work also some DFT test calculations, in particular with the M06 and M06-L exchange-correlation functionals. Both are part of the recently introduced Minnesota 2006 suite of exchange-correlation functionals. ${ }^{33-35}$ M06-L is a local functional, while M06 is a hybrid functional which was parametrized including both transition metals and nonmetals. Both Minnesota functionals include also the density of electron kinetics energy, as characteristic of so-called metaGGA functionals. ${ }^{36}$ In an extensive series of test calculations, these two functionals were shown to perform well for organometallic and inorganometallic thermochemistry. ${ }^{35}$ In this work, their performance for spin-state energetics was tested, both for the three small complexes and for the more realistic models $\mathrm{FeP}(\mathrm{SH})$ and $\mathrm{FeP}(\mathrm{Im})$. More traditional functionals were also employed for comparison, including some common hybrid (B3LYP and B3LYP*), ${ }^{5,37}$ pure (BP86, OLYP), ${ }^{38-40}$ meta-GGA (TPSS), ${ }^{36}$ and hybrid-metaGGA (TPSSh) ${ }^{36}$ functionals.

\section{Computational Details}

All $\mathrm{CASSCF}^{41} / \mathrm{CASPT}^{42,43}$ and some DFT (Minnesota 2006 class of functionals ${ }^{33-35}$ ) calculations were performed with the MOLCAS 7.4 package $^{27}$ using a Cholesky decomposition technique ${ }^{44}$ for approximating the two-electron integrals, with the convergence threshold set to $10^{-6}$ au. DFT calculations for the other functionals were performed with Gaussian $03^{45}$ (B3LYP, B3LYP*, OLYP, BP86, TPSS) or Gaussian $09^{46}$ (TPSSh). In all calculations scalar relativistic effects were included via the second-order Douglas-KrollHess transformation. ${ }^{47}$ All DFT calculations were done using the spin-unrestricted formalism. In all CASPT2 calculations, an imaginary level shift of 0.1 au was used to improve convergence and avoid intruder states. These calculations were performed with either the default IPEA shift of 0.25 au or an increased shift of 0.5 au. Core electrons were kept frozen during the CASPT2 step. For the small heme models, the $\mathrm{Fe}(3 \mathrm{~s}, 3 \mathrm{p})$ electrons were also not included in the correlation, in order to compare to the CASPT2 results to the $\operatorname{CCSD}(\mathrm{T})$ results from Oláh and Harvey, ${ }^{25}$ which did not include these electrons either. For the larger models the $\mathrm{Fe}(3 \mathrm{~s}, 3 \mathrm{p})$ electrons were included, so as to to be able to compare the results obtained here to our previous CASPT2 results for $\mathrm{FeP}(\mathrm{Im}){ }^{23}$

For all five model complexes (Figure 1) single-point CASPT2 and DFT calculations were performed on the B3PW91-optimized geometries from ref 25 (where they were used for the CCSD(T) calculations). Two different types of basis sets were used. The first, correlation-consistent type basis sets, is the same as those used previously for the CCSD(T) calculations. They consist of the Douglas - Kroll recontraction $^{48}$ of the cc-pVDZ, cc-pVTZ, and cc-pVQZ basis sets by Dunning et al. ${ }^{49}$ for $\mathrm{H}, \mathrm{C}, \mathrm{N}, \mathrm{O}$, and $\mathrm{S}$ atoms, combined with cc-pVTZ and cc-pVQZ basis sets for iron, developed by Balabanov and Peterson, also in forms adapted for use with Douglas - Kroll one-electron integrals. ${ }^{50}$ Three combinations were used for iron and the ligand atoms, labeled $\mathrm{A}=$ cc-pVTZ/cc-pVDZ, B = cc-pVTZ/cc-pVTZ, 
Table 1. Number of Contracted Functions Included in Each of the Basis Sets Used in This Work

\begin{tabular}{|c|c|c|c|c|c|}
\hline basis & $\mathrm{Fe}$ & $\mathrm{N}$ & C & $\mathrm{H}$ & $S$ \\
\hline$A=c c-p V T Z / c c-p V D Z$ & $7 s 6 p 4 d 2 f 1 g$ & $3 s 2 p 1 d$ & $3 s 2 p 1 d$ & $2 s 1 p$ & $4 s 3 p 1 d$ \\
\hline $\mathrm{B}=\mathrm{cc}-\mathrm{pVTZ} / \mathrm{cc}-\mathrm{pVTZ}$ & $7 s 6 p 4 d 2 f 1 g$ & $4 s 3 p 2 d 1 f$ & $4 s 3 p 2 d 1 f$ & $3 s 2 p 1 d$ & $5 s 4 p 2 d 1 f$ \\
\hline$C=c c-p V Q Z / c c-p V D Z$ & $8 s 7 p 5 d 3 f 2 g 1 h$ & $3 s 2 p 1 d$ & $3 s 2 p 1 d$ & $2 s 1 p$ & $4 s 3 p 1 d$ \\
\hline II = ANO-rcc/ANO-rcc & $7 s 6 p 5 d 3 f 2 g 1 h$ & $4 s 3 p 2 d 1 f$ & $4 s 3 p 1 d$ & $3 s 1 p$ & $5 s 4 p 3 d 2 f$ \\
\hline $\mathrm{III}=\mathrm{ANO}-\mathrm{rcc} / \mathrm{ANO}-\mathrm{rcc}$ & 10s9p8d6f4g2h & $5 s 4 p 3 d 2 f 1 g$ & $4 s 3 p 2 d 1 f$ & $3 s 2 p 1 d$ & $6 s 5 p 4 d 3 f 2 g$ \\
\hline
\end{tabular}

Table 2. Relative Energy $(\mathrm{kcal} / \mathrm{mol})$ of the Low- and Intermediate-Spin States with Respect to the High-Spin State of the Small Heme Models $1\left({ }^{6} A^{\prime}\right), 2\left({ }^{6} A^{\prime}\right)$, and $3\left({ }^{5} A^{\prime \prime}\right)$ from $\operatorname{CCSD}(T)$ and CASPT2 Calculations

\begin{tabular}{|c|c|c|c|c|c|c|c|c|c|c|c|c|}
\hline & & \multicolumn{4}{|c|}{$\operatorname{CcsD}(T)^{a}$} & \multicolumn{7}{|c|}{ CASPT2 } \\
\hline & & A & B & C & $\infty$ & A & B & C & 1 & II & III & $I I^{b}$ \\
\hline 1 & ${ }^{2} A^{\prime}$ & 37.8 & 38.5 & 33.2 & 29.8 & 35.3 & 35.8 & 30.4 & 33.0 & 30.6 & 30.1 & 25.7 \\
\hline \multirow[t]{2}{*}{2} & ${ }^{2} A^{\prime \prime}$ & 0.6 & & -4.1 & -7.4 & 0.6 & 1.6 & -4.7 & -1.2 & -3.1 & -4.7 & -8.5 \\
\hline & ${ }^{4} A^{\prime \prime}$ & 7.4 & & 4.3 & 2.2 & 6.9 & 7.3 & 3.7 & 5.5 & 4.1 & 3.3 & 1.9 \\
\hline 3 & ${ }^{1} A^{\prime}$ & 37.2 & & 32.9 & 30.4 & 40.2 & 40.3 & 36.1 & 38.6 & 37.3 & 35.9 & 33.8 \\
\hline
\end{tabular}

${ }^{a}$ Taken from ref $25 .{ }^{b}$ IPEA shift of 0.5 au.

and $\mathrm{C}=$ cc-pVQZ/cc-pVDZ. Calculations were also performed with a second type of atomic natural orbital (ANO) basis sets. In these sets, labeled I-III, ANO-rcc basis sets on iron were combined with either ANO-s (basis I) or ANOrcc (basis II, III) on the other atoms. ${ }^{51,52}$ All basis set contractions are given in Table 1. All six basis set combinations were used for the calculations on the small models $\mathbf{1 - 3}$. Calculations on the two larger models, $\mathrm{FeP}(\mathrm{SH})$ and $\mathrm{FeP}(\mathrm{Im})$, were only performed with basis $\mathrm{C}$ and II.

The active space used in the CASSCF and CASPT2 calculations was constructed by starting from a distribution of 6 or 5 electrons in the five Fe $3 d$ orbitals and adding a second $3 \mathrm{~d}^{\prime}$ shell to describe the double-shell effect. ${ }^{53}$ To account for nondynamic correlation effects associated with covalent $\mathrm{Fe}$-ligand interactions, a doubly occupied bonding $\sigma\left(\mathrm{Fe}-\mathrm{N}_{\text {ring }}\right)$ orbital is added, with an additional bonding $\sigma$ $\left(\mathrm{Fe}-\mathrm{NH}_{3}\right.$ ) orbital for model 3 and $\mathrm{FeP}(\mathrm{Im})$, leading to a total of 10 electrons in 12 orbitals, and two additional bonding $\sigma$ (Fe-SH) and $\pi(\mathrm{Fe}-\mathrm{SH})$ orbitals for models 1, 2, and $\mathrm{FeP}(\mathrm{SH})$, leading to a total of 11 electrons in 13 orbitals. In cases where orbitals from the $3 d^{\prime}$ shell correlating empty $3 d$ orbitals were found to rotate out of the active space, they were removed. The empty $3 \mathrm{~d}$ orbitals themselves were kept active and also maintained their character during the orbital optimization process.

\section{Results and Discussion}

3.1. Small Heme Models 1, 2, and 3. All three models consist of an iron atom surrounded by two bidentate amidine ligands, each bonded to iron by 2 nitrogen atoms, and a third axial ligand, either the sulfur-bonded $\mathrm{SH}$ (Fe $\mathrm{Fe}^{\mathrm{III}}$ complexes 1 and 2) or the nitrogen-bonded $\mathrm{NH}_{3}$ ( $\mathrm{Fe}^{\mathrm{II}}$ complex 3) ligand. Interaction with these ligands gives rise to a splitting of the $\mathrm{Fe} 3 \mathrm{~d}$ orbitals, forming pairs of bonding-antibonding molecular orbitals: $\sigma, \sigma^{*}\left(\mathrm{Fe} \mathrm{d}_{x y}-\mathrm{N} ; \mathrm{Fe} \mathrm{d}_{z^{2}}-\mathrm{NH}_{3} / \mathrm{SH}\right)$ and $\pi, \pi^{*}\left(\mathrm{Fe} \mathrm{d}_{y z}-\mathrm{SH}\right)$. The lowest states for each of the different spin multiplicities are ${ }^{6} \mathrm{~A}^{\prime},{ }^{4} \mathrm{~A}^{\prime \prime}$, and ${ }^{2} \mathrm{~A}^{\prime}$ for model $1,{ }^{6} \mathrm{~A}^{\prime}$, ${ }^{4} \mathrm{~A}^{\prime \prime}$, and ${ }^{2} \mathrm{~A}^{\prime \prime}$ for model 2 , and ${ }^{5} \mathrm{~A}^{\prime \prime},{ }^{3} \mathrm{~A}^{\prime}$, and ${ }^{1} \mathrm{~A}^{\prime}$ for model 3. The principal CASSCF configurations (occupations of $\mathrm{Fe}$
$3 \mathrm{~d}$ orbitals) for these states are given in the Supporting Information.

The CCSD(T), CASPT2, and DFT relative energies for the different spin states of the three small heme models are collected in Table 2 for each of the basis sets. Since the DFT results for different basis sets were very similar, only the values of basis set C (cc-pVQZ/cc-pVDZ on iron/ligand) are included in the discussion.

As can be seen from Table 2, all relative energies obtained from either $\operatorname{CCSD}(\mathrm{T})$ and CASPT2 using the same basis sets $(\mathrm{A}-\mathrm{C})$ are in close agreement, with absolute differences below $3 \mathrm{kcal} / \mathrm{mol}$. This is particularly the case for the $\mathrm{Fe}(\mathrm{III})$ model 2, with differences less than $1 \mathrm{kcal} / \mathrm{mol}$ for all states/ basis sets. The same is also true for the ${ }^{4} A^{\prime \prime}-{ }^{6} A^{\prime}$ energy difference in the smaller Fe(III) model 1. On the other hand, the low-spin state ${ }^{2} \mathrm{~A}^{\prime \prime}$ in this case seems to be overstabilized by about $2 \mathrm{kcal} / \mathrm{mol}$ by CASPT2 (as compared to CCSD(T)). This is opposed to the results obtained for the Fe(II) model 3, for which CASPT2 systematically favors the high-spin ${ }^{5} \mathrm{~A}^{\prime}$ ground state, giving rise to energy differences which are larger by $2-3 \mathrm{kcal} / \mathrm{mol}$ than the corresponding $\operatorname{CCSD}(\mathrm{T})$ results.

Table 2 also includes an extrapolation of the $\operatorname{CCSD}(\mathrm{T})$ results to the infinite basis set limit, based on the results obtained with basis A and $\mathrm{C}^{25}$ The choice of only these two sets was based on the observation that the quality of the basis set on iron (cc-pVQZ in basis $C$ versus cc-pVTZ in basis $\mathrm{A}$, combined with the same basis sets on the amidines) influences the spin-state energies of model $\mathbf{1}$ to a much larger extent than the size of the basis set on the amidine ligands (cc-pVTZ in B versus cc-pVDZ in A, combined with the same basis set on iron). The same is also observed for the CASPT2 results for all three models. Between basis $\mathrm{A}$ and $\mathrm{C}$, the relative energies of the highand intermediate-spin states systematically improve by 3-5 $\mathrm{kcal} / \mathrm{mol}$. On the other hand, going from basis A to basis B has a much smaller, and opposite, effect.

In a previous study we made use of ANO-type basis sets, contracted as in basis I and II, to study the bonding of $\mathrm{CO}$, 
Table 3. Relative Energy $(\mathrm{kcal} / \mathrm{mol})$ of the Low- and Intermediate-Spin States with Respect to the High-Spin State of the Small Heme Models $1\left({ }^{6} A^{\prime}\right), 2\left({ }^{6} A^{\prime}\right)$, and $3\left({ }^{5} A^{\prime \prime}\right)$ from DFT Calculations (basis C)

\begin{tabular}{|c|c|c|c|c|c|c|c|c|c|c|}
\hline & & B3LYP & B3LYP* & OLYP & BP86 & TPSS & TPSSh & M06 & M06-L & $\operatorname{ccsD}(T)(\infty)$ \\
\hline 1 & ${ }^{4} A^{\prime \prime}$ & 6.4 & 3.5 & 6.5 & -4.8 & -4.1 & 1.3 & 19.0 & 16.8 & 13.9 \\
\hline 2 & ${ }^{2} A^{\prime \prime}$ & -5.8 & -12.2 & -12.4 & -31.1 & -29.4 & -17.8 & 14.9 & 3.7 & -7.4 \\
\hline \multirow[t]{2}{*}{3} & ${ }^{1} A^{\prime}$ & 26.3 & 22.3 & 27.2 & 12.6 & 12.1 & 20.0 & 35.9 & 27.1 & 30.4 \\
\hline & ${ }^{3} A^{\prime}$ & 10.1 & 7.0 & 9.1 & -1.8 & -2.1 & 4.1 & 21.0 & 15.9 & 17.3 \\
\hline
\end{tabular}

$\mathrm{NO}$, and $\mathrm{O}_{2}$ to $\mathrm{Fe}(\mathrm{II})$ heme systems. ${ }^{23}$ So as to be able to compare the results from that study with the present results, the CASPT2 calculations on models $\mathbf{1}-\mathbf{3}$ were also performed with the same basis sets. Furthermore, the smaller size of the present models also allows us to extend the contraction of the ANO-rcc basis sets even further, thus giving basis III (see Table 1). The size of the contracted basis set I is comparable to basis sets A and B (actually the ANO contraction on iron contains one more $d$ function than the cc-pVTZ set). Still, as Table 2 indicates, the relative energies obtained with basis I are superior to these correlation-consistent sets, predicting, for example, the correct ${ }^{2} \mathrm{~A}^{\prime \prime}$ ground state for model $\mathbf{2}$. The ANO-rcc basis sets II and III contain many more functions on the ligands, yet for the metal they are comparable (either slightly smaller or larger) to ccpVQZ. The fact that these three basis sets give similar relative energies is another confirmation that the ligand basis set size is of minor importance for the property at hand. The results obtained with basis III should be close to the basis set limit for this ANO-rcc basis set on iron. Still, with respect to the $\operatorname{CCSD}(\mathrm{T})$ infinite basis set limit, the CASPT2 results in Table 2 are invariably too high, indicating that in all cases the high-spin state is overstabilized with respect to the lowand intermediate-spin states. This is primarily a basis set effect, pointing to the need of extremely large basis sets on the metal, both primitive and contracted, for an accurate description of the relative spin-state energetics in transitionmetal complexes.

The difference between the best CASPT2 results and the $\operatorname{CCSD}(\mathrm{T})(\infty)$ results is largest for the Fe(II) heme model 3: 4-6 kcal/mol. Here, it should partly be traced back to an inherent tendency of CASPT2 to overstabilize higher with respect to lower spin states in ferrous complexes. Let us note that a comparable error (at least $5 \mathrm{kcal} / \mathrm{mol}$, not accounting for ZPVE) was found in our previous study of the ${ }^{5} \mathrm{~A}_{1 g}-{ }^{3} \mathrm{~A}_{2 g}$ splitting in the four-coordinate FeP complex, calculated there with basis II $^{23}$ and in our recent study of the doublet-quartet transition in some $\{\mathrm{FeNO}\}^{7}$ complexes. ${ }^{54}$ Similar errors were also found in a recent CASPT2 study of the adiabatic quintet-singlet splitting in a number of ferrous pseudooctahedral $\mathrm{FeN}_{6}$ complexes. ${ }^{32}$ On the basis of the results of their study the authors proposed replacing the standard IPEA shift of 0.25 au in the zeroth-order Hamiltonian by a larger value, $0.50-0.70 \mathrm{au}$, for these specific adiabatic gap calculations. In order to investigate whether their proposal can be made more general also for the ferrous and ferric complexes considered here, we decided to repeat the CASPT2 calculations with basis III using an IPEA shift of 0.5 au. The results are given in the rightmost column of the CASPT2 data in Table 2. As one can see, the success of the shift operation is not unequivocal. For the Fe(II) model $\mathbf{3}$, the relative energies are indeed shifted toward the $\operatorname{CCSD}(\mathrm{T})$ results. However, the effect of the IPEA shift increase is too limited, only 0.8 $\mathrm{kcal} / \mathrm{mol}$ for the ${ }^{3} \mathrm{~A}^{\prime}$ and $2.1 \mathrm{kcal} / \mathrm{mol}$ for the ${ }^{1} \mathrm{~A}^{\prime}$ state. The latter value is only about one-half of what was found for the series of seven $\mathrm{FeN}_{6}$ complexes studied in ref 32, showing a systematic increase of their adiabatic HS-LS splitting with $3.6-4.3 \mathrm{kcal} / \mathrm{mol}$ with an increase of the IPEA shift to $0.5 \mathrm{au}$, and further with $3.3-3.8 \mathrm{kcal} / \mathrm{mol}$ with a further increase to $0.75 \mathrm{au}$ On the other hand, for the ferric models 1 and 2, where the original CASPT2 results proved to be excellent, the effect of increasing IPEA is significantly larger than for model 3, up to $4.4 \mathrm{kcal} / \mathrm{mol}$ for the ${ }^{2} \mathrm{~A}^{\prime}$ state, but it is obviously deteriorating. This set of results, although limited, seems to indicate that changing the IPEA shift in the CASPT2 $\hat{H}^{(0)}$ should be done with care, if at all, as the lack of systematics in the approach may easily turn CASPT2 into a semiempirical method.

Turning next to the DFT results (given in Table 3) we first note that, since for this method basis set convergence should be much faster than for traditional correlated ab initio methods, basis $\mathrm{C}$ should be large enough to provide results close to the basis set limit. The DFT results in Table 3 should therefore rather be confronted with the infinite basis set $\operatorname{CCSD}(\mathrm{T})$ results rather than with the results obtained with basis $C$. The $\operatorname{CCSD}(T)(\infty)$ results are therefore included as a reference in the rightmost column of Table 3. A first look at this table already shows that all DFT results significantly differ from the $\operatorname{CCSD}(T)(\infty)$ results. Notably, none of the tested functionals yields an agreement better than $\pm 5 \mathrm{kcal} /$ mol for all three models simultaneously, not rarely the errors exceeding $10 \mathrm{kcal} / \mathrm{mol}$. The DFT energetics are substantially dependent on the functional, in a qualitative agreement with a trend already recognized in the literature: pure functionals systematically overstabilizing low-spin relative to high-spin states, and the hybrid functionals favoring high spins more as the contribution of Hartree-Fock exchange is increased. $5,16,17,19,20,22,23$ Herein, this trend is most clearly evidenced by comparing the TPSS (pure) with the TPSSh (hybrid) results or the B3LYP* (hybrid, 15\% of exact exchange) with the B3LYP results (hybrid, 20\%). The (metaGGA) TPSS functional in fact behaves in a very similar way as the BP86 functional. In contrast, the OLYP functional yields significantly different spin-state energetics than the traditional pure functionals (here epitomized by BP86), in agreement with previous observations. ${ }^{23,55}$ In fact, the present OLYP results are similar to B3LYP* or B3LYP ones. Going to the recently introduced Minnesota functionals (M06 and M06-L) one can note that they both predict a much higher energy of the IS state (with respect to the HS state) than 
Table 4. Relative Energy ( $\mathrm{kcal} / \mathrm{mol})$ of the Low- and Intermediate-Spin States with Respect to the High-Spin State of the Large Heme Models $\mathrm{FeP}(\mathrm{SH})\left({ }^{6} \mathrm{~A}^{\prime}\right)$ and $\mathrm{FeP}(\mathrm{Im})\left({ }^{5} \mathrm{~A}^{\prime}\right)$ from CASPT2 Calculations

\begin{tabular}{ccrrrrrr}
\hline & & \multicolumn{6}{c}{ CASPT2 } \\
\cline { 3 - 7 } basis & \multicolumn{1}{c}{$\mathrm{C}$} & \multicolumn{1}{c}{$\mathrm{I}$} & \multicolumn{1}{c}{$\mathrm{C}^{a}$} & \multicolumn{1}{c}{$\mathrm{I}^{a}$} & $\mathrm{I}^{b}$ & $\mathrm{I}^{c}$ \\
\hline $\mathrm{FeP}(\mathrm{SH})$ & ${ }^{2} \mathrm{~A}^{\prime \prime}$ & 7.0 & 6.8 & 4.1 & 3.6 & & \\
& ${ }^{4} \mathrm{~A}^{\prime \prime}$ & 10.2 & 9.2 & 8.7 & 7.5 & & \\
$\mathrm{FeP}(\mathrm{Im})$ & ${ }^{1} \mathrm{~A}^{\prime}$ & 11.2 & 10.6 & 14.4 & 13.7 & 14.0 & 13.0 \\
& ${ }^{3} \mathrm{~A}^{\prime \prime}$ & 10.3 & 9.5 & 10.3 & 9.5 & 8.6 & 8.5
\end{tabular}

${ }^{a}$ With $\mathrm{Fe}(3 \mathrm{~s}, 3 \mathrm{p})$ core electron correlation included. ${ }^{b}$ From ref 23 using PBEO structures (and omitting the ZPVE contribution). ${ }^{c}$ From ref 23 using BP86 structures (and omitting the ZPVE contribution).

any other of the tested functionals; the same holds true for the energy of the LS state (with respect to the HS state), except for model 3 for M06-L. Two more observations can be made for the Minnesota functionals. First, the local functional M06-L in general performs better than the M06 functional. The latter functional systematically overstabilizes the high-spin with respect to the intermediate-spin and even more with respect to the low-spin state, with errors amounting up to $22 \mathrm{kcal} / \mathrm{mol}$. A second observation is that the ferric complexes, with model $\mathbf{2}$ in particular, are described considerably worse than the ferrous complex $\mathbf{3}$. For the latter model, quite reasonable results are in fact obtained with both functionals, M06 overestimating the relative energies by $2-4$ $\mathrm{kcal} / \mathrm{mol}$ and M06-L underestimating them with $1-3 \mathrm{kcal} /$ mol. In contrast, for model $\mathbf{2}$, both functionals severely overestimate the stability of the high-spin ${ }^{6} \mathrm{~A}^{\prime}$ state, incorrectly predicting this state to be the ground state. With M06, the ordering of the two other states ${ }^{2} \mathrm{~A}^{\prime \prime}$ and ${ }^{4} \mathrm{~A}^{\prime \prime}$ is also not correctly reproduced.

3.2. Large Heme Models FeP(SH) and FeP(Im). Interaction of the iron atom with the surrounding porphyrin and axial ligand gives rise to a splitting of the d orbitals similar to that of the small model complexes, thus forming pairs of bonding-antibonding molecular orbitals: $\sigma, \sigma^{*}\left(\mathrm{Fe} \mathrm{d}_{x y}-\mathrm{P}\right.$; Fe $\left.\mathrm{d}_{z^{2}}-\mathrm{Im} / \mathrm{SH}\right)$ and $\pi, \pi^{*}\left(\mathrm{Fe} \mathrm{d}_{x z}-\mathrm{SH}\right)$. The lowest states for each spin are ${ }^{6} \mathrm{~A}^{\prime},{ }^{4} \mathrm{~A}^{\prime \prime}$, and ${ }^{2} \mathrm{~A}^{\prime \prime}$ for $\mathrm{FeP}(\mathrm{SH})$ and ${ }^{5} \mathrm{~A}^{\prime}$, ${ }^{3} \mathrm{~A}^{\prime \prime}$, and ${ }^{1} \mathrm{~A}^{\prime}$ for $\mathrm{FeP}(\mathrm{Im})$. The relative energies of the different spin states are presented in Table 4.

Compared to the small models $\mathbf{1}$ and $\mathbf{3}$, the CASPT2 relative energies of the LS and IS states of $\mathrm{FeP}(\mathrm{SH})$ and $\mathrm{FeP}(\mathrm{Im})$ are much lower, though the HS state remains the ground state for both of the large heme models. The ${ }^{2} \mathrm{~A}^{\prime \prime}$ state of $\mathrm{FeP}(\mathrm{SH})$ is lower in energy than the ${ }^{4} \mathrm{~A}^{\prime \prime}$ state, but unlike in model 2 it remains above the ${ }^{6} \mathrm{~A}^{\prime}$ state. The relative energies of the ${ }^{1} \mathrm{~A}^{\prime}$ and ${ }^{3} \mathrm{~A}^{\prime \prime}$ states of $\mathrm{FeP}(\mathrm{Im})$ are similar, about $10 \mathrm{kcal} / \mathrm{mol}$ above the ${ }^{5} \mathrm{~A}^{\prime}$ ground state. Going from basis $\mathrm{C}$ to the ANO basis set II, an increased stabilization of the low- and intermediate-spin states is observed for both complexes. The same basis set was used by Radon and Pierloot $^{23}$ to calculate the relative energies of the different spin states of FeP and FeP(Im), using the same active space but different geometries, that is PBE0 and BP86 optimized and $\mathrm{Fe}(3 \mathrm{~s}, 3 \mathrm{p})$ core correlation. The results for $\mathrm{FeP}(\mathrm{Im})$ were added to Table 4. In order to compare with these results, extra CASPT2 calculations with $\mathrm{Fe}(3 \mathrm{~s}, 3 \mathrm{p})$ core correlation were performed for basis $\mathrm{C}$ and II. We can see that the energies are very similar to the values obtained here using the B3PW91-optimized structures from ref 25. Both singlet and triplet excited-state energies are within a range of 0.5 $\mathrm{kcal} / \mathrm{mol}$ around 13.5 and $9.0 \mathrm{kcal} / \mathrm{mol}$, respectively. We also note that the effect of $\mathrm{Fe}(3 \mathrm{~s}, 3 \mathrm{p})$ correlation is rather significant (to about $3 \mathrm{kcal} / \mathrm{mol}$ ) and opposite for both complexes: in FeP(Im) the LS and IS states are stabilized with respect to the HS state, while in $\mathrm{FeP}(\mathrm{SH})$ the LS state is destabilized and the IS state is unaffected. In view of these irregularities we believe that the $3 \mathrm{~s}, 3 \mathrm{p}$ electrons should be preferably correlated in ab initio calculations of spin-state energetics in first-row transition-metal complexes.

Going to the DFT results (Table 5), we first note that they compare well to the previous DFT calculations on the heme complexes $(\mathrm{FeP}(\mathrm{Im})$ and $\mathrm{FeP}(\mathrm{SH}))$ available in the literature. ${ }^{23,25,56-59}$ As could be expected, a similar behavior of the different DFT functionals is found for the large heme models as for the small models $\mathbf{1 - 3}$. In this respect we note again that the energies of the LS and IS states (with respect to the HS state) are lower with the pure (BP86, TPSS) than with the hybrid functionals (B3LYP, B3LYP *,TPSSh), with OLYP giving results close to the hybrid functionals. A more concrete discussion of the accuracy of the DFT results for $\mathrm{FeP}(\mathrm{Im})$ and $\mathrm{FeP}(\mathrm{SH})$ requires a comparison to some reliable reference results. A reasonable estimate of the spin splitting in these complexes may be obtained from the available CASPT2 results (Table 4), assuming that this method has similar errors for the large models as were noted for models 1-3. Our "best" estimate of the splittings is given in the rightmost column of Table 5. As one can see, the results of the hybrid functionals (B3LYP, B3LYP*) and the pure OLYP functional are reasonably close to this estimate, with errors typically $\leq 6 \mathrm{kcal} / \mathrm{mol}$. The results obtained from the pure TPSS and BP86 functionals are worse and again (see also Table 3) very similar, overstabilizing the IS and LS states by $12-16 \mathrm{kcal} / \mathrm{mol}$ with respect to the HS state for $\mathrm{FeP}(\mathrm{SH})$ and by $16-20 \mathrm{kcal} / \mathrm{mol}$ for $\mathrm{FeP}(\mathrm{Im})$. The error is reduced in TPSSh; however, somewhat suprisingly, this method now overshoots the relative energy of the ${ }^{4} \mathrm{~A}^{\prime \prime}$ state in $\mathrm{FeP}(\mathrm{SH})$.

A more important question is whether M06 or M06-L can outperform the traditional functionals. This is obviously not the case. Similar to the small models, we find that both functionals tend to overstabilize the HS with respect to the IS and even more with respect to the LS state. The M06-L functional again clearly outperforms M06. As was also found for models 1-3 both Minnesota functionals describe the ferrous complex FeP(Im) much better than the ferric complex $\mathrm{FeP}(\mathrm{SH})$. In fact, for the former (ferrous) complex, the M06-L functional yields quite accurate spin-state energetics. This success should however be put into perspective, given the much larger error of more than $10 \mathrm{kcal} / \mathrm{mol}$ obtained with this functional for the ${ }^{2} \mathrm{~A}^{\prime \prime}$ state in $\mathrm{FeP}(\mathrm{SH})$.

\section{Conclusion}

In this investigation we attempted to benchmark the accuracy of CASPT2 and selected DFT methods for spin-state energetics of selected heme complexes of $\mathrm{Fe}(\mathrm{II})$ and $\mathrm{Fe}(\mathrm{III})$, 
Table 5. Relative Energy $(\mathrm{kcal} / \mathrm{mol})$ of the Low- and Intermediate-Spin States with Respect to the High-Spin State of the Large Heme Models FeP(SH) $\left({ }^{6} \mathrm{~A}^{\prime}\right)$ and $\mathrm{FeP}(\mathrm{Im})\left({ }^{5} \mathrm{~A}^{\prime}\right)$ from DFT Calculations with Basis $\mathrm{C}$

\begin{tabular}{|c|c|c|c|c|c|c|c|c|c|c|}
\hline & & B3LYP & B3LYP* & OLYP & BP86 & TPSS & TPSSh & M06 & M06-L & "best" estimate ${ }^{a}$ \\
\hline $\mathrm{FeP}(\mathrm{SH})$ & ${ }^{2} A^{\prime \prime}$ & 3.8 & -1.2 & 1.9 & -16.3 & -15.8 & -6.1 & 24.1 & 14.5 & $-0.7-3.5$ \\
\hline & ${ }^{4} A^{\prime \prime}$ & 2.0 & -1.0 & 2.6 & -9.5 & -8.2 & 10.3 & 14.9 & 12.0 & $4.2-7.2$ \\
\hline $\mathrm{FeP}(\mathrm{Im})$ & ${ }^{1} A^{\prime}$ & 6.5 & 1.1 & 4.2 & -14.4 & -13.1 & -2.5 & 17.7 & 5.6 & $6.8-8.7$ \\
\hline
\end{tabular}

${ }^{a}$ CASPT2 results from Table 4, corrected for the errors found for the small models $1-\mathbf{3}$. The "best" estimates were obtained by adding to the CASPT2 results (including $(3 \mathrm{~s}, 3 \mathrm{p})$ correlation) the difference between the CASPT2 and $\operatorname{CCSD}(\mathrm{T})(\infty)$ calculations for the corresponding small models. When using either the results from basis $\mathrm{C}$ and II and either models $\mathbf{1}$ or 2 for FeP(SH) the range of values given in the rightmost column is obtained.

including the models of the active site of cytochrome P450 (in its resting state) and myoglobin. While there are no benchmark results for the large heme complexes ( $\mathrm{FeP}(\mathrm{Im})$ and $\mathrm{FeP}(\mathrm{SH})$ ), the $\mathrm{CCSD}(\mathrm{T})$ calculations for their smaller mimics (complexes $\mathbf{1}-\mathbf{3}$ ) were recently published. ${ }^{25}$ It must be mentioned here that although we believe in a high accuracy of the reference $\operatorname{CCSD}(\mathrm{T})$ data, one should not forget that they also might be subject to errors related to the absence of higher order terms in the CC expansion, the multiconfigurational character of the wave function, or the basis set extrapolation procedure.

The performance of CASPT2 is excellent for the ferric complexes $\mathbf{1}$ and $\mathbf{2}$ (an error within chemical accuracy) and worse for the ferrous complex $\mathbf{3}$, thereby confirming previous suspicions that this method overstabilizes the high-spin state in some $\mathrm{Fe}(\mathrm{II})$ complexes. $^{23,32,54}$ Let us note that all these problematic cases concern the ligand-field transitions from a nonbonding $\left(\mathrm{d}_{x^{2}-y^{2}}\right)$ to an antibonding $\left(\mathrm{d}_{x y}\right)$ orbital of $\mathrm{Fe}$. The error can be estimated as slightly above $5 \mathrm{kcal} / \mathrm{mol}$ but definitely less than $10 \mathrm{kcal} / \mathrm{mol}$. It should be stressed that CASPT2 errors of this size are rather exceptional in transition-metal chemistry, even for $\mathrm{d}_{x^{2}-y^{2}} \rightarrow \mathrm{d}_{x y}$ transitions. This is illustrated by the excellent performance of this method for complexes $\mathbf{1}$ and $\mathbf{2}$ as well as by previous numerous applications. Unfortunately, it seems that for the presently studied complexes the error cannot be easily reduced by changing the zeroth-order Hamiltonian of CASPT2 (increasing the IPEA shift), as was successful in the previous study of some $\mathrm{FeN}_{6}$ complexes. This indicates that playing with the zeroth-order Hamiltonian of CASPT2 should be done with care (and preferably avoided); otherwise, one may easily turn this ab initio method into a de facto semiempirical approach.

The present investigation also explored the accuracy of several DFT methods. With respect to the extrapolated $\operatorname{CCSD}(\mathrm{T})$ reference results, all tested functionals lead to errors above $5 \mathrm{kcal} / \mathrm{mol}$ at least for one complex. This is also true for the recently introduced M06 and M06-L functionals from the Minnesota 2006 set, among which the second one (M06-L) performs much better. However, its overall performance for the complexes studied here is not any better than of some more traditional functionals, like B3LYP or OLYP.

In summary, the present investigation confirms an overall high accuracy of CASPT2, although a systematic error of CASPT2 for the ferrous complexes (possibly one of the most difficult cases for CASPT2 calculations) is definitely pinpointed. We believe that CASPT2 calculations on transition- metal systems will become more and more common and useful in the field of bioinorganic chemistry.

Acknowledgment. This investigation was supported by grants from the Flemish Science Foundation (FWO) and the Concerted Research Action of the Flemish Government (GOA) and by the Polish State Ministry of Science and Higher Education (MNiSW) from the funds for scientific research (2009-2010). The computational grant from the Academic Computer Center CYFRONET AGH is also acknowledged.

Supporting Information Available: Plots of the active orbitals for the small models $\mathbf{1}$ and $\mathbf{3}$ and the large models $\mathrm{FeP}(\mathrm{Im})$ and $\mathrm{FeP}(\mathrm{SH})$; occupation numbers of the $\mathrm{Fe} 3 \mathrm{~d}$ orbitals in the lowest state of each spin and symmetry in the different models; graphical representation of the differences obtained with different methods for the (IS-HS) and (LSHS) splittings with respect to $\operatorname{CCSD}(T)(\infty))$. This material is available free of charge via the Internet at http:// pubs.acs.org.

\section{References}

(1) In Iron Porphyrins; Lever, A. B. P., Gray, H. B., Eds.; Addison-Wesley: Reading, MA, 1983.

(2) In The Porphyrin Handbook; Kadish, K., Smith, K., Guillard, R., Eds.; Academic Press: New York, 2000.

(3) Kozlowski, P. M.; Spiro, T. G.; Bérces, A.; Zgierski, M. Z. J. Phys. Chem. B 1998, 102, 2603-2608.

(4) Paulsen, H.; Duelund, L.; Winkler, H.; Toftlund, H.; Trautwein, A. X. Inorg. Chem. 2001, 40, 2201-2203.

(5) Reiher, M.; Salomon, O.; Hess, B. A. Theor. Chem. Acc. 2001, 107, 48-55.

(6) Reiher, M. Inorg. Chem. 2002, 41, 6928-6935.

(7) Salomon, O.; Reiher, M.; Hess, B. A. J. Chem. Phys. 2002, 117, 4729-4737.

(8) Baranović, G. Chem. Phys. Lett. 2003, 369, 668-672.

(9) Ghosh, A.; Vangberg, T.; Gonzalez, E.; Taylor, P. R. J. Porphyrins Phthalocyanins 2001, 5, 345-356.

(10) Ghosh, A.; Persson, B. J.; Taylor, P. R. J. Biol. Inorg. Chem. 2003, 8, 507-511.

(11) Ghosh, A.; Tangen, E.; Ryeng, H.; Taylor, P. R. Eur. J. Inorg. Chem. 2004, 4555-4560.

(12) Paulsen, H.; Trautwein, A. X. Top. Curr. Chem. 2004, 235, 197-219.

(13) Harvey, J. N. Struct. Bonding (Berlin) 2004, 112, 151-183. 
(14) Deeth, R. J.; Fey, N. J. Comput. Chem. 2004, 25, 18401848.

(15) Swart, M.; Groenhof, A. R.; Ehlers, A. W.; Lammertsma, K. J. Phys. Chem. A 2004, 108, 5479-5483.

(16) Fouqueau, A.; Casida, S. M. M. E.; Daku, L. M. L.; Hauser, A.; Neese, F. J. Chem. Phys. 2004, 120, 9473-9486.

(17) Fouqueau, A.; Casida, M. E.; Daku, L. M. L.; Hauser, A.; Neese, F. J. Chem. Phys. 2005, 122, 044110.

(18) Daku, L. M. L.; Vargas, A.; Hauser, A.; Fouqueau, A.; Cassida, M. E. Chem. Phys. Chem. 2005, 6, 1393-1410.

(19) Ganzenmüller, G.; Berkane, N.; Fouqueau, A.; Casida, M. E. J. Chem. Phys. 2005, 122, 234321.

(20) Pierloot, K.; Vancoillie, S. J. Chem. Phys. 2006, 125, 124303.

(21) Strickland, N.; Harvey, J. N. J. Phys. Chem. B 2007, 111, 841-852.

(22) Pierloot, K.; Vancoillie, S. J. Chem. Phys. 2008, 128, 034104.

(23) Radoń, M.; Pierloot, K. J. Phys. Chem. A 2008, 112, 1182411832.

(24) Khvostichenko, D.; Choi, A.; Boulatov, R. J. Phys. Chem. A 2008, 112, 3700-3711.

(25) Oláh, J.; Harvey, J. N. J. Phys. Chem. A 2009, 113, 73387345.

(26) Pierloot, K. Mol. Phys. 2003, 101, 2083-2094.

(27) Karlström, G.; Lindh, R.; Malmqvist, P.-Å.; Roos, B. O.; Ryde, U.; Veryazov, V.; Widmark, P.-O.; Cossi, M.; Schimmelpfennig, B.; Neogrady, P.; Seijo, L. Comput. Mater. Sci. 2003, 28, 222-239.

(28) Andersson, K.; Roos, B. O. Int. J. Quantum Chem. 1993, 45, 591-607.

(29) Roos, B. O.; Andersson, K.; Fülscher, M. P.; Malmqvist, P.Å.; Serrano-Andrés, L.; Pierloot, K.; Merchán, M. Multiconfigurational Perturbation Theory: Applications in Electronic Spectroscopy. In Advances in Chemical Physics: New Methods in Computational Quantum Mechanics; Prigogine, I., Rice, S. A., Eds.; John Wiley \& Sons: New York, 1996; Vol. XCIII, pp 219-332.

(30) Andersson, K. Theor. Chim. Acta 1995, 91, 31-46.

(31) Ghigo, G.; Roos, B. O.; Malmqvist, P.-Å. Chem. Phys. Lett. 2004, 396, 142-149.

(32) Kepenekian, M.; Robert, V.; Le Guennic, B. J. Chem. Phys. 2009, 131, 114702.

(33) Zhao, Y.; Truhlar, D. G. J. Chem. Phys. 2006, 125, 194101.

(34) Zhao, Y.; Truhlar, D. G. J. Phys. Chem. A 2006, 110, 1312613130.

(35) Zhao, Y.; Truhlar, D. G. Theor. Chem. Acc. 2008, 120, 215241.
(36) Tao, J.; Perdew, J. P.; Staroverov, V. N.; Scuseria, G. E. Phys. Rev. Lett. 2003, 91, 146401-146404.

(37) Becke, A. D. J. Chem. Phys. 1993, 98, 5648-5652.

(38) Becke, A. D. Phys. Rev. A 1988, 38, 3098-3100.

(39) Perdew, J. P. Phys. Rev. B 1986, 33, 8822-8824.

(40) Handy, N. C.; Cohen, A. J. Mol. Phys. 2001, 99, 403-412.

(41) Malmqvist, P.-Å.; Rendell, A.; Roos, B. O. J. Phys. Chem. 1990, 94, 5477-5482.

(42) Andersson, K.; Malmqvist, P.-Å.; Roos, B. O.; Sadlej, A. J.; Wolinski, K. J. Chem. Phys. 1990, 94, 5483.

(43) Andersson, K.; Malmqvist, P.-Å.; Roos, B. O. J. Chem. Phys. 1992, 96, 1218.

(44) Aquilante, F.; Pedersen, T. B.; Lindh, R. J. Chem. Phys. 2007, 126,194106

(45) Frisch, M. J. Gaussian 03, Revision C.02; Gaussian Inc., Wallingford, CT, 2004.

(46) Frisch, M. J. Gaussian 09, Revision A.02; Gaussian Inc.: Wallingford, CT, 2009.

(47) Reiher, M.; Wolf, A. J. Chem. Phys. 2004, 121, 10945-10956.

(48) de Jong, W. A.; Harrison, R. J.; Dixon, D. A. J. Chem. Phys. 2001, 114, 48-53.

(49) Dunning, T. H. J. Chem. Phys. 1989, 90, 1007-1023.

(50) Balabanov, N. B.; Peterson, K. A. J. Chem. Phys. 2005, 123, 064107.

(51) Roos, B. O.; Lindh, R.; Malmqvist, P.-Å.; Veryazov, V.; Widmark, P.-O. J. Phys. Chem. A 2005, 109, 6575-6579.

(52) Pierloot, K.; Dumez, B.; Widmark, P.-O.; Roos, B. O. Theor. Chem. Acc. 1995, 90, 87.

(53) Andersson, K.; Roos, B. O. Chem. Phys. Lett. 1992, 191, 507-514.

(54) Radoń, M.; Broclawik, E.; Pierloot, K. J. Phys. Chem. B 2010, in press.

(55) Conradie, J.; Ghosh, A. J. Phys. Chem. B 2007, 111, 1262112624.

(56) Groenhof, A. R.; Swart, M.; Ehlers, A. W.; Lammertsma, K. J. Phys. Chem. 2005, 109, 3411-3417.

(57) Shaik, S.; Kumar, D.; de Visser, S. P.; Altun, A.; Thiel, W. Chem. Rev. 2005, 105, 2279-2328.

(58) Rydberg, P.; Sigfridsson, E.; Ryde, U. J. Biol. Inorg. Chem. 2004, 9, 203-223.

(59) Strickland, N.; Harvey, J. N. J. Phys. Chem. B 2007, 111, 841-852.

CT900567C 